PROCEEDINGS OF THE

AMERICAN MATHEMATICAL SOCIETY

Volume 134, Number 12, December 2006, Pages 3507-3513

S 0002-9939(06)08482-6

Article electronically published on June 27, 2006

\title{
CONFORMAL MAPPING AND ELLIPSES
}

\author{
ARNE LEDET AND ALEXANDER YU. SOLYNIN
}

(Communicated by Juha M. Heinonen)

\begin{abstract}
We answer a question raised by M. Chuaqui, P. Duren, and B. Osgood by showing that a conformal mapping of a simply connected domain cannot take two circles onto two proper ellipses.
\end{abstract}

The goal of this note is to prove the following.

Proposition 1. Let $\Omega$ be a simply connected domain on $\overline{\mathbb{C}}=\mathbb{C} \cup\{\infty\}$ containing circles (on $\overline{\mathbb{C}}$ ) $C_{1}$ and $C_{2}, C_{1} \neq C_{2}$. Let $f$ be a conformal mapping from $\Omega$ into $\mathbb{C}$. If $f$ maps each of the circles $C_{1}$ and $C_{2}$ onto an ellipse, then $f$ is a Möbius transformation and therefore the ellipses are actually circles.

Thus this proposition answers affirmatively a question raised by M. Chuaqui, P. Duren, and B. Osgood in [1 that a conformal mapping of a simply connected domain cannot take two circles onto two ellipses.

Precomposing $f$ with a Möbius transformation and postcomposing it with a linear transformation, we may assume that $C_{1}=\{z:|z|=1\}$ and that $f$ maps the unit disc $\mathbb{D}=\{z:|z|<1\}$ onto the interior of an ellipse $\mathcal{L}_{1}$ with foci \pm 1 such that $f(r)=1, f(-r)=-1$ for some $0<r<1$. Then it is a standard exercise in complex analysis to verify that $w=f(z)$ is defined by

(1) $w=\frac{1}{2}(\zeta+1 / \zeta) \quad$ with $\quad \zeta=i \exp \left\{-\frac{\pi i}{2 \mathbf{K}\left(r^{2}\right)} \int_{0}^{z / r} \frac{d t}{\sqrt{\left(1-t^{2}\right)\left(1-r^{4} t^{2}\right)}}\right\}$,

where $\mathbf{K}(\cdot)$ denotes the complete elliptic integral of the first kind. Since the image $f(\mathbb{D})$ is symmetric with respect to the coordinate axes, it follows that $f$ is an odd function having real coefficients, i.e.,

$$
f(z)=p z+c_{3} z^{3}+c_{5} z^{5}+\ldots \quad \text { with } p=\pi /\left(2 r \mathbf{K}\left(r^{2}\right)\right)>0 .
$$

We want to stress that our proof below does not require the explicit form (1) of the mapping function, which is included here for completeness.

Instead, we will use the fact that every ellipse $\mathcal{L}$ having foci $w_{1}$ and $w_{2}$ is a trajectory of the quadratic differential

$$
Q_{1}(w) d w^{2}=-\frac{d w^{2}}{\left(w-w_{1}\right)\left(w-w_{2}\right)} ;
$$

Received by the editors June 6, 2005 .

2000 Mathematics Subject Classification. Primary 30C35.

Key words and phrases. Conformal mapping, ellipse.

The research of the second author was supported in part by NSF grant DMS-0412908.

(C)2006 American Mathematical Society 
we refer to [2] for the definition and necessary properties of quadratic differentials. Let $w=f(z)$ map a disc $D$ on $\overline{\mathbb{C}}$ conformally onto the interior of $\mathcal{L}$. Let $z_{1}$ and $z_{2}$ be the preimages in $D$ of $w_{1}$ and $w_{2}$ and let $z_{3}, z_{4}$ be the reflections of $z_{1}, z_{2}$ in the circle $C=\partial D$. Pulling the quadratic differential (2) back to the disc $D$ we obtain a quadratic differential $Q_{2}(z) d z^{2}=Q_{1}(f(z)) f^{\prime 2}(z) d z^{2}$ positive on $C$ whose only singularities in $D$ are simple poles at $z_{1}$ and $z_{2}$. The latter implies that

$$
Q_{2}(z) d z^{2}=-k \frac{d z^{2}}{\left(z-z_{1}\right)\left(z-z_{2}\right)\left(z-z_{3}\right)\left(z-z_{4}\right)},
$$

with some nonzero $k \in \mathbb{C}$. In (3), we assume that all singularities are finite. If one of them, say $z_{j}$, is infinite, the corresponding term $\left(z-z_{j}\right)$ in (3) must be replaced by 1 .

Comparing (2) and (3) we find that the mapping $w=f(z)$ from $D$ onto the interior of $\mathcal{L}$ satisfies the differential equation

$$
{w^{\prime}}^{2}=k \frac{\left(w-w_{1}\right)\left(w-w_{2}\right)}{\left(z-z_{1}\right)\left(z-z_{2}\right)\left(z-z_{3}\right)\left(z-z_{4}\right)} .
$$

For a particular choice when $D=\mathbb{D}, w_{1}=1, w_{2}=-1, z_{1}=r$, and $z_{2}=-r$ with $0<r<1$, (4) becomes

$$
{w^{\prime}}^{2}=-p^{2} \frac{\left(w^{2}-1\right)}{\left(z^{2}-r^{2}\right)\left(z^{2}-1 / r^{2}\right)}, \quad \text { where } p=w^{\prime}(0)>0 .
$$

Now we assume that $\Omega$ is a simply connected domain on $\overline{\mathbb{C}}$ containing the discs $\mathbb{D}$ and $D \neq \mathbb{D}$. If there is a function $w=f(z)$ univalent in $\Omega$, which maps the circles $C_{1}=\partial \mathbb{D}$ and $C_{2}=\partial D$ onto ellipses, then, by the principle of analytic continuation, $w=f(z)$ will satisfy near the origin both equations (4) and (5). Therefore the Taylor coefficients of $f$ at $z=0$ can be found from either one of these equations.

To simplify our computation, we first take logarithmic derivatives in (4) and (5), then expand them into power series at $z=0$. For our purposes we need the first eight coefficients, so all our computations can be easily verified with Maple or Mathematica. Equating the first eight Taylor coefficients of the results and assuming that $z_{1}, z_{2} z_{3}, z_{4}, w_{1}, w_{2}$ all are nonzero and finite we obtain in this generic case the following system of eight polynomial equations, which we call system $(*)$ :

$$
\begin{aligned}
a+b+c+d= & p(A+B), \\
a^{2}+b^{2}+c^{2}+d^{2}= & p^{2}\left(A^{2}+B^{2}\right)+2\left(\left(r^{2}+1 / r^{2}\right)-p^{2}\right), \\
a^{3}+b^{3}+c^{3}+d^{3}= & p^{3}\left(A^{3}+B^{3}\right)+\frac{1}{2} p(A+B)\left(\left(r^{2}+1 / r^{2}\right)-p^{2}\right), \\
a^{4}+b^{4}+c^{4}+d^{4}= & p^{4}\left(A^{4}+B^{4}\right)+\frac{2}{3} p^{2}\left(A^{2}+B^{2}-2\right)\left(\left(r^{2}+1 / r^{2}\right)-p^{2}\right) \\
& +2\left(\left(r^{4}+1 / r^{4}\right)-p^{4}\right), \\
a^{5}+b^{5}+c^{5}+d^{5}= & p^{5}\left(A^{5}+B^{5}\right)+\frac{1}{4} p(A+B)+\frac{3}{8} p(A+B)\left(\left(r^{4}+1 / r^{4}\right)-p^{4}\right) \\
& +\frac{5}{12} p^{3}\left[2\left(A^{3}+B^{3}\right)-(A+B)\right]\left(\left(r^{2}+1 / r^{2}\right)-p^{2}\right), \\
a^{6}+b^{6}+c^{6}+d^{6}= & p^{6}\left(A^{6}+B^{6}\right)+2\left(\left(r^{6}+1 / r^{6}\right)-p^{6}\right)+\frac{7}{15} p^{2}\left(A^{2}+B^{2}-2\right) \\
& +\frac{8}{15} p^{2}\left(A^{2}+B^{2}-2\right)\left(\left(r^{4}+1 / r^{4}\right)-p^{4}\right) \\
& +p^{4}\left[\left(A^{4}+B^{4}\right)-\frac{2}{3}\left(A^{2}+B^{2}+1\right)\right]\left(\left(r^{2}+1 / r^{2}\right)-p^{2}\right),
\end{aligned}
$$




$$
\begin{aligned}
a^{7}+b^{7}+c^{7}+d^{7}= & p^{7}\left(A^{7}+B^{7}\right)+\frac{5}{16} p(A+B)\left(\left(r^{6}+1 / r^{6}\right)-p^{6}\right) \\
+ & \frac{259}{720} p^{3}\left[2\left(A^{3}+B^{3}\right)-(A+B)\right]\left(\left(r^{4}+1 / r^{4}\right)-p^{4}\right) \\
+ & \left\{\frac{7}{144} p^{5}\left[24\left(A^{5}+B^{5}\right)-20\left(A^{3}+B^{3}\right)+(A+B)\right]\right. \\
& \left.+\frac{3}{16} p(A+B)\right\}\left(\left(r^{2}+1 / r^{2}\right)-p^{2}\right) \\
& +\frac{133}{180} p^{3}\left[\left(A^{3}+B^{3}\right)-(A+B)\right]+\frac{401}{720} p^{3}(A+B), \\
a^{8}+b^{8}+c^{8}+d^{8}= & p^{8}\left(A^{8}+B^{8}\right)+2\left(\left(r^{8}+1 / r^{8}-p^{8}\right)\right) \\
& +\frac{16}{35} p^{2}\left(A^{2}+B^{2}-2\right)\left(\left(r^{6}+1 / r^{6}\right)-p^{6}\right) \\
& +\frac{14}{45} p^{4}\left[3\left(A^{4}+B^{4}\right)-2\left(A^{2}+B^{2}\right)-2\right]\left(\left(r^{4}+1 / r^{4}\right)-p^{4}\right) \\
& +p^{6}\left[\frac{4}{3}\left(\left(A^{6}+B^{6}\right)-\left(A^{4}+B^{4}\right)\right)\right. \\
& \left.+\frac{8}{45}\left(A^{2}+B^{2}\right)-\frac{16}{45}\right]\left(\left(r^{2}+1 / r^{2}\right)-p^{2}\right) \\
& +\frac{8}{21} p^{2}\left(A^{2}+B^{2}-2\right)\left(\left(r^{2}+1 / r^{2}\right)-p^{2}\right),
\end{aligned}
$$

where $a=1 / z_{1}, b=1 / z_{2}, c=1 / z_{3}, d=1 / z_{4}, A=1 / w_{1}$, and $B=1 / w_{2}$.

From this point, our argument is purely algebraic. Our goal is to show that the only solution to system $(*)$ is $a=r, b=-r, c=1 / r, d=-1 / r$ (up to permutation), and $A=1, B=-1$ (again up to permutation).

Since the left-hand sides of the equations in system $(*)$ are Newton power sums in $a, b, c$ and $d$, they are, in particular, symmetric polynomials, and can therefore be written in terms of the elementary symmetric symbols $s_{1}=a+b+c+d, \ldots$, $s_{4}=a b c d$. For necessary properties of the Newton power sums and symmetric polynomials, one may consult [3, Section 5.7]. Similarly, $A$ and $B$ only occur on the right-hand sides of system $(*)$ in the form of Newton power sums, which can be expressed in terms of the elementary symmetric symbols $S_{1}=A+B$ and $S_{2}=A B$. The goal is then to show that the only solution to the rewritten system is $s_{1}=0$, $s_{2}=-\left(r^{2}+1 / r^{2}\right), s_{3}=0, s_{4}=1, S_{1}=0$, and $S_{2}=-1$.

The first equation of system $(*)$ now takes the form $s_{1}=p S_{1}$, which determines $s_{1}$. The second equation becomes

$$
s_{1}^{2}-2 s_{2}=p^{2}\left(S_{1}^{2}-2 S_{2}\right)+2\left(\left(r^{2}+1 / r^{2}\right)-p^{2}\right),
$$

which we can solve to find

$$
s_{2}=p^{2} S_{2}-\left(\left(r^{2}+1 / r^{2}\right)-p^{2}\right) .
$$

The third equation is

$$
s_{1}^{3}-3 s_{1} s_{2}+3 s_{3}=p^{3}\left(S_{1}^{3}-3 S_{1} S_{2}\right)+3 q S_{1},
$$

and we solve this to find

$$
s_{3}=-\frac{5 p S_{1}}{6}\left(\left(r^{2}+1 / r^{2}\right)-p^{2}\right) .
$$

The fourth equation becomes

$$
\begin{aligned}
& s_{1}^{4}+4 s_{1} s_{3}-4 s_{1}^{2} s_{2}+2 s_{2}^{2}-4 s_{4} \\
& \quad=4 p q\left(S_{1}^{2}-2 S_{2}\right)+p^{4}\left(S_{1}^{4}-4 S_{1}^{2} S_{2}+2 S_{2}^{2}\right)-8 p q+2\left(\left(r^{4}+1 / r^{4}\right)-p^{4}\right),
\end{aligned}
$$


which we solve to find

$$
s_{4}=1-\frac{2}{3} p^{2}\left(S_{2}+1\right)\left(\left(r^{2}+1 / r^{2}\right)-p^{2}\right) .
$$

This determines $s_{1}, s_{2}, s_{3}, s_{4}$ as functions of $S_{1}$ and $S_{2}$. Also, for $S_{1}=0$ and $S_{2}=-1$, this gives the required values for $s_{1}, s_{2}, s_{3}, s_{4}$. Thus, the only thing remaining is to show that $S_{1}=0$ and $S_{2}=-1$ is the only solution to the last four equations of system $(*)$.

The fifth equation becomes

$$
p S_{1}\left(61 p^{4}+100 p^{2}-50 p^{2} R^{2}-11 R^{4}+44 R^{2}+64\right)=0,
$$

where $R=r+1 / r$. Therefore since $p \neq 0$ by (5), we must either have $S_{1}=0$ or

$$
61 p^{4}+100 p^{2}-50 p^{2} R^{2}-11 R^{4}+44 R^{2}+64=0 .
$$

Now, assume $S_{1} \neq 0$. Then (6) is satisfied.

The sixth equation of system $(*)$ written in terms of symmetric symbols $S_{1}$ and $S_{2}$ becomes

$$
\begin{aligned}
60 p^{2}\left[3 \left(61 p^{4}+\right.\right. & \left.100 p^{2}-50 p^{2} R^{2}-11 R^{4}+44 R^{2}+64\right) S_{1}^{2} \\
& \left.+8\left(-8-28 R^{2}+7 R^{4}-20 p^{2}+10 p^{2} R^{2}-17 p^{4}\right)\left(S_{2}+1\right)\right]=0 .
\end{aligned}
$$

So assuming (6), we must in fact have

$$
\left(-8-28 R^{2}+7 R^{4}-20 p^{2}+10 p^{2} R^{2}-17 p^{4}\right)\left(S_{2}+1\right)=0 .
$$

Hence, $S_{2}=-1$ or

$$
-8-28 R^{2}+7 R^{4}-20 p^{2}+10 p^{2} R^{2}-17 p^{4}=0 .
$$

In the second case, we obtain

$$
\begin{aligned}
& \left(61 p^{4}+100 p^{2}-50 p^{2} R^{2}-11 R^{4}+44 R^{2}+64\right) \\
& +5\left(-8-28 R^{2}+7 R^{4}-20 p^{2}+10 p^{2} R^{2}-17 p^{4}\right) \\
& =24\left(R^{4}-4 R^{2}+\left(1-p^{4}\right)\right)=0
\end{aligned}
$$

meaning that

$$
R=\sqrt{2 \pm \sqrt{3+p^{4}}}
$$

Plugging this into (66), we get

$$
25\left(3+2 p^{4} \pm 2 p^{2} \sqrt{3+p^{4}}\right)=0,
$$

which is easily seen to be impossible. Therefore, we must have $S_{2}=-1$.

The seventh equation of system $(*)$ can be written as

$$
\begin{aligned}
& p S_{1}\left[42\left(61 p^{4}+100 p^{2}-50 p^{2} R^{2}-11 R^{4}+44 R^{2}+64\right) p^{2} S_{1}^{2}\right. \\
& +\left(1246 R^{4}-4984 R^{2}+3220 p^{2} R^{2}-3584-6440 p^{2}-4466 p^{4}\right) p^{2} S_{2} \\
& +\left(5355 p^{4} R^{2}+3690 R^{4}-2040 R^{2}-615 R^{6}-3081 p^{6}-12264 p^{2}+6636 p^{2} R^{2}\right. \\
& \left.\left.\quad-10710 p^{4}-1659 p^{2} R^{4}-5760\right)\right]=0
\end{aligned}
$$


which simplifies to

$$
-2\left(49 p^{4}+44\right) R^{2}+98 p^{6}+196 p^{4}+235 p^{2}+176=0,
$$

when we use (6) and $S_{2}=-1$. Consequently,

$$
R=\sqrt{\frac{98 p^{6}+196 p^{4}+235 p^{2}+176}{2\left(49 p^{4}+44\right)}},
$$

and (6) becomes

$$
\frac{1089\left(768+637 p^{4}\right)}{4\left(49 p^{4}+44\right)^{2}}=0,
$$

which is impossible as well, since $p$ is real.

All in all, we must therefore have that $S_{1}=0$. Again, (7) reduces to

$$
\left(-8-28 R^{2}+7 R^{4}-20 p^{2}+10 p^{2} R^{2}-17 p^{4}\right)\left(S_{2}+1\right)=0,
$$

so if $S_{2}$ does not equal -1 , we have

$$
-8-28 R^{2}+7 R^{4}-20 p^{2}+10 p^{2} R^{2}-17 p^{4}=0 .
$$

Now, the eighth equation of system $(*)$ is

$$
\begin{aligned}
\left(1+S_{2}\right)\left[-7\left(-8-28 R^{2}+\right.\right. & \left.7 R^{4}-20 p^{2}+10 p^{2} R^{2}-17 p^{4}\right) p^{2} S_{2} \\
+\left(132+378 p^{2}-\right. & 252 p^{2} R^{2}+486 R^{2}+63 p^{2} R^{4} \\
- & \left.\left.189 p^{4} R^{2}-414 R^{4}+378 p^{4}+57 p^{6}+69 R^{6}\right)\right]=0
\end{aligned}
$$

and so assuming (9),

$132+378 p^{2}-252 p^{2} R^{2}+486 R^{2}+63 p^{2} R^{4}-189 p^{4} R^{2}-414 R^{4}+378 p^{4}+57 p^{6}+69 R^{6}=0$.

Taking this times 49 , adding $-483 R^{2}+996+249 p^{2}$ times (9), and dividing the result by 90 , this reduces to

$$
\left(16 p^{4}+7\right) R^{2}-\left(16 p^{6}+32 p^{4}+31 p^{2}+14\right)=0,
$$

i.e.,

$$
R=\sqrt{\frac{16 p^{6}+32 p^{4}+31 p^{2}+14}{16 p^{2}+7}} .
$$

Substituting this into (9), we get

$$
-\frac{1764}{\left(16 p^{4}+7\right)^{2}}=0
$$

This is of course absurd, so we conclude that $S_{2}=-1$.

Thus, $S_{1}=0$ and $S_{2}=-1$ is the only solution, and the proposition is proved in the generic case.

Turning to the remaining special cases, we note that if $z_{k}=\infty$, then the corresponding term $a, b, c$, or $d$ in the left-hand side of system $(*)$ is absent, which does not effect further computation.

Next, since $f(z)$ is analytic at $z=0$, it follows from (44) that $z_{k}=0$ for some $k$ if and only if $w_{1}=0$ or $w_{2}=0$. Assume $w_{1}=0$. Then also $z_{1}=0$ since $z_{1}$ denotes the preimage of $w_{1}$. Now we can of course find equations similar to those 
of system $(*)$, where the left-hand side of the equations are Newton power sums in $b=1 / z_{2}, c=1 / z_{3}$, and $d=1 / z_{4}$, and the right-hand side are expressions in $r$, $p$ and $B=1 / w_{2}$. In this case, having six variables, we need the first six equations. Again, the Newton power sums are rewritten in terms of the elementary symmetric symbols $s_{1}=b+c+d, s_{2}=b c+b d+c d$, and $s_{3}=b c d$, and the first three equations are used to solve for $s_{1}, s_{2}$, and $s_{3}$ in terms of $r, p$, and $B$. As a further simplification, we replace $r+1 / r$ by $R$, to get the remaining three equations

$$
\begin{aligned}
& L_{4}:=11 R^{4}+\left(50 p^{2}-44\right) R^{2}-\left(61 p^{4}+100 p^{2}+64\right)=0, \\
& L_{5}:=\left(24 R^{4}-\left(70 p^{2}+92\right) R^{2}+\left(47 p^{4}+140 p^{2}+128\right)\right) B=0, \\
& \begin{aligned}
L_{6}:=- & 7 p^{2}\left(24 R^{4}-\left(70 p^{2}+92\right) R^{2}+\left(47 p^{4}+140 p^{2}+128\right)\right) B^{2} \\
& \quad+269 R^{6}+\left(1085 p^{2}-1614\right) R^{4}+\left(936-4340 p^{2}-1729 p^{4}\right) R^{2} \\
& \quad+\left(375 p^{6}+3458 p^{4}+4760 p^{2}+2432\right)=0 .
\end{aligned}
\end{aligned}
$$

Here, we note an obvious simplification: $L_{6}+7 p^{2} B L_{5}$ does not depend on $B$, but is simply a polynomial in $R$ and $p$. Furthermore,

$$
\begin{aligned}
\frac{1}{480}\left(121\left(L_{6}+7 p^{2} B L_{5}\right)+\right. & \left.\left(5918+1515 p^{2}-2959 R^{2}\right) L_{4}\right) \\
& =2\left(49 p^{2}+44\right) R^{2}-\left(98 p^{6}+196 p^{4}+235 p^{2}+176\right)=0,
\end{aligned}
$$

so we must have

$$
R=\sqrt{\frac{98 p^{6}+196 p^{4}+235 p^{2}+176}{2\left(49 p^{2}+44\right)}} .
$$

But then

$$
L_{4}=-\frac{1089\left(637 p^{4}+768\right)}{4\left(49 p^{2}+44\right)^{2}},
$$

which cannot be zero, since $p$ is real.

Therefore, there are no solutions in this case. This finishes the proof of Proposition 1.

Remarks. (1) Actually our proof gives a stronger result: Let $f$ admit analytic continuation along any path in a domain (not necessarily simply connected) $\Omega \subset \overline{\mathbb{C}}$. Suppose there are two branches $f_{1}$ and $f_{2}$ of $f$ and two discs $D_{1}$ and $D_{2}, D_{1} \neq D_{2}$, such that $f_{k}$ maps $D_{k}$ one-to-one onto an interior of an ellipse. Then $f$ is a Möbius transformation.

(2) Equating the right-hand sides of (4) and (5) we can express $w$ as an algebraic function of $z$. Then one may finish the proof of Proposition 1 by invoking the fact that the elliptic integral in (11) cannot be reduced to an algebraic form for any value of $r$ under consideration. But we could not find a reference for such a result in the accessible literature.

(3) It is worth mentioning that the existence of a common solution $w=f(z)$ to two equations $w^{\prime 2}=R_{1}(z, w)$ and $w^{\prime 2}=R_{2}(z, w)$ with rational $R_{1}$ and $R_{2}$ does not guarantee in general that $R_{1} \equiv R_{2}$. Indeed, the Koebe function $w=$ $z /(1-z)^{2}$, being extremal in numerous problems of geometric function theory, satisfies a variety of such equations; see [2, Ch. VI]. 


\section{REFERENCES}

1. M. Chuaqui, P. Duren, and B. Osgood, Ellipses, near ellipses, and harmonic Möbius transformations. Proc. Amer. Math. Soc., 133 (2005), no. 9, 2705-2710. MR2146217(2005m:31002)

2. J. A. Jenkins, Univalent functions and conformal mapping, 2nd ed. Springer, Berlin, 1965. MR.0096806 (20:3288)

3. B. L. van der Waerden, Algebra. Vol. I. Springer-Verlag, New York, 1991. MR1080172 (91h:00009a)

Department of Mathematics and Statistics, Texas Tech University, Box 41042, LubBOCK, TEXAS 79409

E-mail address: arne.ledet@ttu.edu

Department of Mathematics and Statistics, Texas Tech University, Box 41042, LubBOCK, TEXAS 79409

E-mail address: alex.solynin@ttu.edu 